\title{
Air Path Dynamics Control and Efficiency Optimization with Scenario Approach for Gasoline Engines
}

\author{
Weihai Jiang ${ }^{1)}$ Tielong Shen ${ }^{1)}$ \\ 1) Sophia University, Department of Engineering of Science \\ 7-1Kioicho Chiyoda-ku.Tokyo 102-8554, Japan (E-mail:weihaijiang@eagle.sophia.ac.jp, tetu-sin@sophia.ac.jp)
}

Received on October 26, 2018

\begin{abstract}
This paper presents a control design approach of efficiency optimization while providing the desired torque under the constraint of combustion variation for gasoline engines with EGR. Mainly, the first phase of control design is the equilibrium point optimization under the chance constraint of IMEP probability distribution with a scenario approach, and the second phase is nonlinear dynamic feedback stabilization control design for improving transient performance of the state regulation. The experimental validations demonstrate that the control scheme can achieve stable combustion and fast transient control performance.
\end{abstract}

KEY WORDS: gasoline engine, EGR, optimization, chance constraint, stabilization control (E1)

\section{Introduction}

Over the past decades, it has been proved that Cooled external Exhaust Gas Recirculation (cEGR) has a large potential to improve the fuel efficiency of gasoline engines and has gained significant attention on both the academic research field and the industry application field ${ }^{(1-5)}$. However, the cooled external EGR causes an unwanted coupling and nonlinear effect on the fresh air charging and combustion ${ }^{(6-8)}$, and results in a significant challenge on the control of the combustion. To make things worse, it may trigger unstable combustion without carefully controlling ${ }^{(1,9)}$. To try to handle this challenge issue, a lot of studies have focused on the sense of mean value model via dynamic system control ${ }^{(6-8)}$. On the sense of the cyclic transient behavior, disturbance rejection-based feedback control ${ }^{(10)}$, MPC based ${ }^{(9)}$, extremum seeking $^{(11)}$, adaptive control ${ }^{(12)}$ etc. have been studied.

However, it should be noted that the cycle based combustion event of the combustion engine is essentially a stochastic phenomenon and highly stochastic ${ }^{(13-15)}$, which makes the control challenging. Unlike the traditional deterministic optimization problems, the constraints of stochastic optimization problem are infinite since the sample space is uncountable. Then, there are infinite possibilities to extract the uncertain constraints ${ }^{(16)}$, and generally result in NP-hard ${ }^{(17-18)}$. This implies that these problems are either not easy solvable or computational hard and conservative by obtaining the worst-case solution via relaxation of the constraint ${ }^{(19)}$.

Fortunately, the stochastic optimization control theory has been widely investigated over the past decades, in which the socalled chance-constraint optimization( $\mathrm{CCO}$ ) has been recognized as an appropriately feasible and robust way to handle the stochastic optimization problem ${ }^{(19-20)}$. The philosophy of CCO is that it transforms the all uncertainty constraint of the original infinite optimal problem into a probability sense based finite constraint problem with a scenario approach ${ }^{(19)}$. Thus, the CCO based control structure may be a prospective and feasible way to achieve the efficiency optimization control of the air path system for gasoline engines under the stochastic combustion variation constraint.

In this paper, a control scheme is proposed for coordinating control of EGR valve and throttle valve in a gasoline engine to achieve the maximum efficiency while providing the desired engine torque with guaranteeing stable combustion based on $\mathrm{CCO}$. To guarantee the stable combustion, the variation of the IMEP is restrained by a stochastic optimization approach to obtain the optimal equilibrium points. Then, the nonlinear feedback controller is designed to achieve the equilibrium points stabilization tracking control. Finally, the effectiveness of proposed control scheme is validated in a 4-cyliner gasoline engine.

\section{Problem description}

\subsection{Physical and modeling}

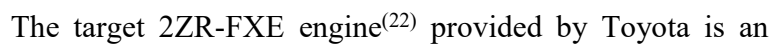
advanced hybrid application engine which is equipped with EGR and intake VVT system. Unlike the traditional EGR system, the EGR gas of this engine is transported to the intake port and distributed into each cylinder directly, which implies there is little mixing effect between the EGR gas and intake air in the intake manifold ${ }^{(22)}$. And, we can assume that the EGR gas always be inducted into the cylinder from the EGR valve. According to the 
ideal gas equation and mass conversation law ${ }^{(1,21)}$ with the assumptions in ${ }^{(22)}$, the intake manifold and exhaust manifold dynamic modeling ${ }^{(22)}$ can be described as following:

$$
\begin{gathered}
\dot{p}_{i m}=\frac{R_{i m} T_{i m}}{V_{i m}}\left(\dot{m}_{t h r}+\dot{m}_{E G R}-\dot{m}_{e i}\right) \\
\dot{p}_{e m}=\frac{R_{e m} T_{e m}}{V_{e m}}\left(\dot{m}_{e i}+\dot{m}_{f}-\dot{m}_{E G R}-\dot{m}_{t w c}\right)
\end{gathered}
$$

where, $p_{i m}, p_{e m}$ in [pa] stands for the intake manifold and exhaust manifold pressure state respectively. R, T, V, $\dot{m}$ denote the ideal gas constant $[\mathrm{J} / \mathrm{kg} \cdot \mathrm{k}]$, temperature $[\mathrm{K}]$, volume $\left[\mathrm{m}^{3}\right]$ and charge mass flow $[\mathrm{kg} / \mathrm{s}]$ in the given equations with the specific subscripts.

The torque modeling ${ }^{(24)}$ is summarized as:

$$
\begin{aligned}
\tau_{e}= & L H V_{f} \dot{m}_{f}\left(1-\frac{1}{r_{e}^{\gamma-1}}\right) \cdot \eta_{E G R}\left(x_{E G R}\right) / 4 \pi \\
& -\frac{V_{d}}{4 \pi}\left(p_{e m}-p_{\text {im }}\right)-f_{\text {fric }}\left(N_{e}\right)
\end{aligned}
$$

where, $x_{E G R}, \eta_{E G R}$ and $f_{\text {fric }}$ are the EGR ratio, efficiency and fraction torque function respectively.

\subsection{Combustion process}

Fig. 1 illustrates the results of the control input EGR valve on the combustion, while the corresponding throttle is adjusted to remain the torque under a fix value. At the same time, the spark advance is controlled at MBT and the fuel is controlled by ECU to guarantee Lambda is stoichiometric.
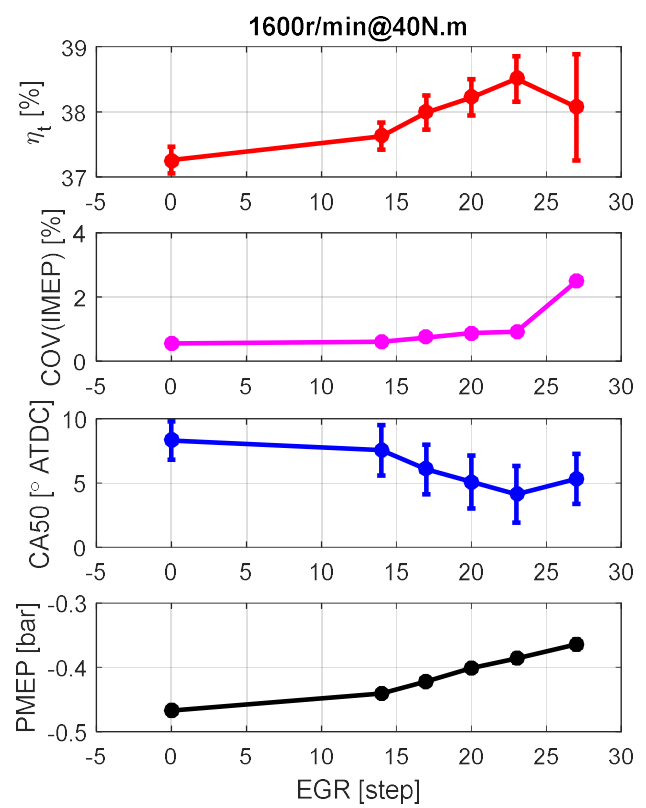

Fig. 1 The influence of EGR on combustion

It is noticed that the pumping loss can be reduced almost linearly with the increasing of the EGR valve, while the corresponding combustion of variation $\left(C_{I M E P}\right)$ will increase very fast along with further increasing of the EGR vale when the $\operatorname{COV}_{I M E P}$ is larger than $2 \%$ of this EGR system, and the thermal efficiency will decrease at the same time. The reason of this phenomenon may be that the spark advance would have less effect on optimizing the combustion, as it is shown that the CA50 is retarded, when using too much EGR and $C O V_{I M E P}$ is larger than $2 \%$. Thus, in this research, we choose $\operatorname{COV}_{I M E P}=2 \%$ as the up boundary of the combustion.

However, due to the combustion process is stochastic, the effect of the EGR on the combustion is uncertain even under the steady state and IMEP distribution under steady condition obeys Gauss distribution ${ }^{[23]}$.

Since the mean value of IMEP is influenced by the fuel mass flow and the thermal efficiency, the mean IMEP modeling can be expressed by the function of the fuel mass flow and thermal efficiency which can be regressed by the EGR ratio and engine speed. Thus, the EGR-IMEP modeling can be expressed as a mean value function coupling with a normal stochastic distribution.

$$
I M E P=\mu_{I M E P}+\varepsilon, \varepsilon \in N\left(0, \sigma^{2}\right)
$$

Here, the mean value model of IMEP $\mu_{I M E P}$ is modeled by a regression model, as shown follows,

$$
\mu_{I M E P}=m_{f} \cdot\left[\left(1-1 /\left(r_{e}^{\gamma-1}\right)\right)\left(a_{1} \cdot x_{E G R}^{2}+a_{2} x_{E G R}+a_{3}\right)+a_{4}\right]
$$

where, $a_{1}, a_{2}, a_{3}$ and $a_{4}$ are the parameters to be identified by experiments.

\subsection{The Analysis of Chance-Constraint of IMEP Variation}

The Coefficient of variance of IMEP, $C O V_{I M E P}$ can be expressed into the following equation:

$$
C O V_{\text {IMEP }}=\frac{\sigma_{I M E P}}{\mu_{I M E P}} \times 100 \%
$$

where $\mu_{I M E P}$ is the expected value, and $\mu_{I M E P}=E\left\{I M E P_{i}\right\}$, $\mathrm{i}=1,2 \ldots \mathrm{N}, \sigma_{I M E P}$ is the standard deviation, and $\sigma_{I M E P}=$ $\sqrt{E\left\{I M E P_{i}-\mu_{I M E P}\right\}}, \mathrm{i}=1,2 \ldots \mathrm{N}$.

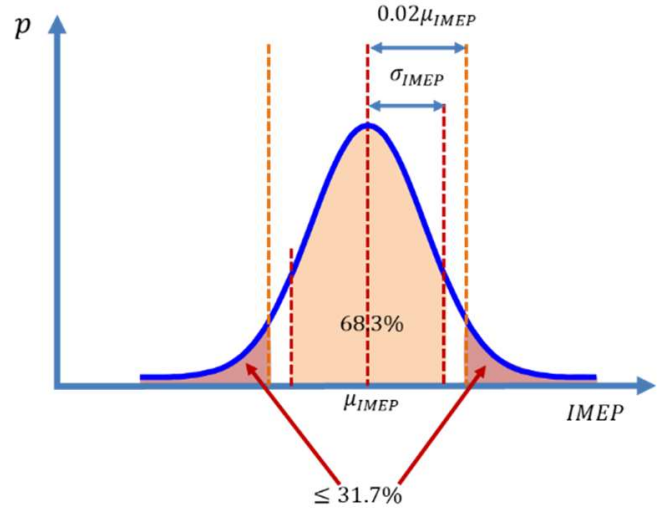

Fig.2 The normal probability distribution characteristic of IMEP

In this research, we limit the up boundary of the $\operatorname{COV}_{I M E P}$ as $2 \%$, which means $\operatorname{COV}_{I M E P} \leq 0.02$. We assumed that the IMEP distribution is a normal distribution, then the relationship of the $\mu_{I M E P}, \sigma_{I M E P}$ and the corresponding probability density function (PDF) can be illustrated in the Fig. 2. For a given PDF, the probability can be computed in a finite interval. And, the $\sigma_{I M E P} \leq 0.02 \mu_{I M E P}$ can be interpreted in a probabilistic perspective, which means the probability of the 
distance between IMEP and the mean value larger than $0.02 \mu_{I M E P}$ should no less than $1 \sigma_{I M E P}$. Then, the constraint $\operatorname{COV}_{I M E P} \leq 0.02$ can be trasformed into the IMEP probability constraint, which is showed in the following inequality.

$$
p\left\{\left|I M E P-\mu_{I M E P}\right|>0.02 \mu_{I M E P}\right\} \leq 31.7 \%
$$

\subsection{Problem formulation}

Engine control problem is to achieve the minimized fuel economy and satisfy the emission constraint and the torque requirement from the driver. Considering pumping loss occupied $7 \%$ of the total energy, then reducing pumping loss have a great potential to improve the fuel efficiency. For gasoline engine with EGR system, this research is focused on minimizing the pumping loss while achieve the desired torque under the constraint of the IMEP distribution constraint.

\section{Control design}

Figure 3 illustrates the general framework of the proposed control structure which is mainly two phases: the optimal equilibrium point design and online nonlinear feedback control.

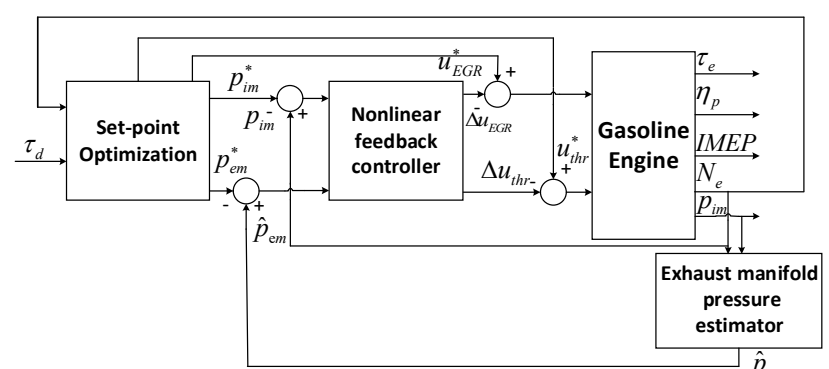

Fig. 3 The general structure of the proposed control scheme

The controller is developed based on the simplified control oriented second order model (1-2). Firstly, the optimal set point of $p_{i m}^{*}$ and $p_{e m}^{*}$ are designed by solving the stationary optimization problem under the IMEP probability chance-constraint and torque requirement. While the stabilization feedback controller based on constructed Lyapunov function is designed to track the optimal equilibrium points i.e. track the optimal state of $p_{i m}^{*}$ and $p_{e m}^{*}$, to improve the transient performance. What's more, considering the exhaust manifold pressure are usually cost prohibitive to measure, the exhaust manifold pressure estimator, which is proposed by the authors' previous research ${ }^{(22)}$, is used here to substitute to the physical sensor.

\subsection{Set-point optimization design with scenario approach}

As for the optimal set-point design, the pumping work is first defined which can be approximated by $\mathrm{V}_{\mathrm{d}}\left(p_{e m}-p_{i m}\right)^{(21)}$, and has been demonstrated to have a good accuracy during most of the operating conditions ${ }^{(21)}$. Then, the cost function of the pumping loss is defined as $\eta_{p}=\mathrm{V}_{\mathrm{d}}\left(p_{e m}-p_{i m}\right)^{(21)}$. Consequently, the multivariable multi-objective optimal control problem can be described as: for a given desired torque demand $\tau_{d}$, minimize the cost function under the IMEP stochastic variation constraint. As analyzed in previous section, the IMEP variation constraint problem can be transformed into the IMEP probability constraint problem. Then, to achieve efficiency optimization under torque requirement and IMEP variation constraint, the optimal vector variables $\left(p_{i m}^{*}, p_{e m}^{*}, u_{t h r}^{*}, u_{E G R}^{*}\right)$ can be obtained by solving the following formulated chance constraint optimization problem, which is defined as the problem $\mathcal{P}$.

$$
\begin{array}{ll}
\mathcal{P}: & \min _{\left\{p_{i m}^{*}, p_{e m}^{*}, u_{h r}^{*}, u_{E G R}^{*}\right\}}-\eta_{\mathrm{p}}\left(p_{i m}, p_{e m}\right) \\
\text { s.t. } & \tau_{d}-\tau_{e}\left(u_{E G R,} p_{i m}, p_{e m}, N_{e}\right)=0 \\
& \operatorname{Pr}\left(\left|I M E P\left(p_{i m}, p_{e m}, u_{E G R}, \delta_{I M E P}\right)-\mu_{I M E P}\right| \geq 0.02 \mu_{I M E P}\right) \leq \alpha \\
& \left(p_{i m}, p_{e m}\right) \in\left[\underline{p}_{i m}, \bar{p}_{i m}\right] \times\left[\underline{p}_{e m}, \bar{p}_{e m}\right] \\
& \left(u_{t h r}, u_{E G R}\right) \in\left[\underline{u}_{t h r}, \bar{u}_{t h r}\right] \times\left[\underline{u}_{E G R}, \bar{u}_{E G R}\right]
\end{array}
$$

where, $\delta_{I M E P} \in \Omega_{I M E P}$ is the stochastic variable of IMEP, and $\Omega_{I M E P}$ represents for the sample space of $\delta_{I M E P}$. The state variable vector $\left(p_{i m}, p_{e m}\right)$ and control input variable vector $\left(u_{t h r}, u_{E G R}\right)$ belong to the admissible set of $\left[\underline{p}_{i m}, \bar{p}_{i m}\right] \times\left[\underline{p}_{e m}, \bar{p}_{e m}\right]$ and $\left[\underline{u}_{t h r}, \bar{u}_{t h r}\right] \times\left[\underline{u}_{E G R}, \bar{u}_{E G R}\right]$ respectively. $\tau_{d}$ is the desired torque. However, since the exist of the probability constraint, it is generally very hard to get an efficient numerical solution of the problem $\mathcal{P}$, and is generally NP-hard ${ }^{(18)}$. The so called "scenario approach"(19) can provide an approximated solution of optimization problem $\mathcal{P}$. Then, the following deterministic optimization problem can be formulated with finite $\mathrm{N}$ of samplings from the sample space through the scenario design, and is defined as problem of $\mathcal{P}_{C C O}$.

$$
\begin{array}{ll}
\mathcal{P}_{C C O}: \min _{\left\{p_{i m}^{*}, p_{e m}^{*}, u_{l t r}^{*}, u_{E G R}^{*}\right\}}-\eta_{\mathrm{p}}\left(p_{i m}, p_{e m}\right) \\
\text { s.t. } \quad \tau_{d}-\tau_{e}\left(\mathrm{u}_{E G R}, \mathrm{p}_{i m}, p_{e m}, N_{e}\right)=0 \\
& \left|\operatorname{IMEP}(\mathrm{i})-\mu_{I M E P}\right| \leq 0.02 \mu_{I M E P}, \mathrm{i} \in 1,2 \ldots N
\end{array}
$$

where, IMEP(i) denotes the $i^{\text {th }}$ sample which is extracted from $\Omega_{I M E P}$. To be mentioned, scenario here means that any realization or instance of the uncertainty parameter $\delta_{M E P}$ being sampled from the space of $\Omega_{I M E P}$. Then, the extract of the scenario i is stochastic and hence the solution of problem (9) is also stochastic. The scenario number $\mathrm{N}$ should also be designed properly in the follows to guarantee the probability feasible of the solution. Then, to guarantee the probability feasibility of the solution $u_{N}$ of problem $\mathcal{P}_{C C O}$, the randomly selected number $\mathrm{N}$ (scenario) in the formulated optimization problem in (9) should be designed according to proposition 1 based on the original problem (8).

Proposition 1: For the given $\alpha$ level, and with $\beta$ probability that the optimal solution of $\mathcal{P}_{C C O}$ is not satisfied of the stochastic constraint in problem $\mathcal{P}$, if the sample number $\mathrm{N}$ satisfies the following inequality, 


$$
N \geq N_{g e n} \doteq\left\lceil\frac{2}{1-\alpha} \ln \frac{1}{\beta}+2 n_{\mu}+\frac{2 n_{\mu}}{1-\alpha} \ln \frac{2}{1-\alpha}\right\rceil \text { (21) }
$$

where, $\lceil\cdot\rceil$ and $n_{\mu}$ denote the smallest integer greater than or equal to the argument and the dimension of $\mu$, respectively. Then, the optimal solution of the scenario problem $\mathcal{P}_{C C O}$ is $\alpha$ level robustly feasible with probability no smaller than $1-\beta$.

Compared with the problem $\mathcal{P}$, the problem $\mathcal{P}_{C C O}$ is a standard optimization problem with only finite $\mathrm{N}$ of constraints, which can be computed by using the various optimal numerical algorithms. What's more, it can be noted that optimal solution of the scenario problem $\mathcal{P}_{C C O}$ outperforms the robust convex problem $\mathcal{P}$ with all the constraints being considered. In this degree, the scenario problem $\mathcal{P}_{C C O}$ alleviates the conservatism of the original stochastic optimal problem $\mathcal{P}$.

\subsection{Nonlinear stabilization feedback controller design}

To achieve the optimal equilibrium point tracking control, as the second control design phase, the Lyapunov-based nonlinear feedback control will be designed based on the nonlinear model.

For the simplicity of notation, we note $r_{i m}=R_{i m} V_{i m} / T_{i m}$, $r_{e m}=R_{e m} V_{e m} / T_{e m}$. Noticing that both the control input have direct control on the intake manifold pressure, to decouple the relationship and simplify the model to make it better for the controller design, the coordinate transformation is made as following,

$$
\left\{\begin{array}{l}
\dot{p}_{\Sigma}=r_{i m}\left(\dot{m}_{t h r}+\dot{m}_{f}-\dot{m}_{t w c}\right) \\
\dot{p}_{e m}=r_{e m}\left(\dot{m}_{e i}+\dot{m}_{f}-\dot{m}_{E G R}-\dot{m}_{t w c}\right)
\end{array}\right.
$$

Considering the control oriented model may exist model error applied in the real system, the model error term $\Delta_{\Sigma}, \Delta_{e m}$ is added as showed in the following equality

$$
\left\{\begin{array}{l}
\dot{p}_{\Sigma}=r_{i m}\left(\dot{m}_{t h r}+\dot{m}_{f}-\dot{m}_{t w c}+\Delta_{\Sigma}\right) \\
\dot{p}_{e m}=r_{e m}\left(\dot{m}_{e i}+\dot{m}_{f}-\dot{m}_{E G R}-\dot{m}_{t w c}+\Delta_{e m}\right)
\end{array}\right.
$$

Then error dynamic function is constructed to design the controller. Firstly, the sigma error function is defined as: $e_{\Sigma}=$ $p_{\Sigma}-p_{\Sigma}^{*}$

Let the throttle mass follow as:

$$
\dot{m}_{t h r}=\dot{m}_{t w c}-\dot{m}_{f}-\mathrm{K}_{1} e_{\Sigma}-\mathrm{K}_{2} \int e_{\Sigma} d t
$$

Then, the sigma error dynamic can be finally formulated as:

$$
\dot{e}_{\Sigma}=r_{m}\left(-\mathrm{K}_{1} e_{\Sigma}-\mathrm{K}_{2} \int e_{\Sigma} d t+\Delta_{\Sigma}\right)
$$

Define the error of sigma model as $v_{\Sigma}=\mathrm{K}_{2} \int e_{\Sigma} d t$, then:

$$
\tilde{v}_{\Sigma}=\Delta_{\Sigma}-\mathrm{K}_{2} \int e_{\Sigma} d t
$$

Then, the sigma error dynamic function can be written as:

$$
\dot{e}_{\Sigma}=r_{m}\left(-\mathrm{K}_{1} e_{\Sigma}+\tilde{v}_{\Sigma}\right)
$$

Define the exhaust manifold error dynamic function $e_{e m}=$ $p_{e m}-p_{e m}^{*}$. And let the EGR mass flow

$$
\begin{gathered}
\dot{m}_{E G R}=\dot{m}_{e i}+\dot{m}_{f}-\dot{m}_{t w c}+\mathrm{K}_{3} e_{e m}+\mathrm{K}_{4} \int e_{e m} d t \\
\dot{e}_{e m}=r_{e m}\left(-\mathrm{K}_{3} e_{e m}-\mathrm{K}_{4} \int e_{e m} d t+\Delta_{e m}\right)
\end{gathered}
$$

Define the exhaust manifold pressure model error as:

$$
\begin{gathered}
v_{e m}=\mathrm{K}_{4} \int e_{e m} d t \\
\tilde{v}_{e m}=\Delta_{e m}-\mathrm{K}_{4} \int e_{e m} d t
\end{gathered}
$$

Then, the $p_{e m}$ error dynamic function can be written as:

$$
\dot{e}_{e m}=r_{e m}\left(-\mathrm{K}_{3} e_{e m}+\tilde{v}_{e m}\right)
$$

Proposition 2: For any given desired equilibrium point $\left(p_{i m}^{*}, p_{e m}^{*}\right)$, let the feedback control law as followings:

$$
\begin{aligned}
& \dot{m}_{t \mathrm{~h} r}=\dot{m}_{t w c}-\dot{m}_{f}-K_{1} e_{\Sigma}-K_{2} \int e_{\Sigma} d t \\
& \dot{m}_{E G R}=\dot{m}_{e i}+\dot{m}_{f}+K_{3} e_{e m}+K_{4} \int e_{e m} d t
\end{aligned}
$$

Then, with the control law (22-23), the closed loop system (16) and (21) is asymptotic stable at the given equilibrium point $\left(p_{i m}^{*}, p_{e m}^{*}\right)$, if the control gains $\mathrm{K} 1, \mathrm{~K} 2, \mathrm{~K} 3, \mathrm{~K} 4$ are all positive, which means $e_{\Sigma}, \tilde{\vartheta}_{\Sigma}, e_{e m}, \tilde{\vartheta}_{e m} \rightarrow 0$ as $\mathrm{t} \rightarrow 0$.

Proof: the error dynamic function can be summarized as,

$$
\left\{\begin{array}{l}
\dot{e}_{\Sigma}=r_{m}\left(-\mathrm{K}_{1} e_{\Sigma}+\tilde{v}_{\Sigma}\right) \\
\dot{\tilde{v}}_{\Sigma}=-\mathrm{K}_{2} e_{\Sigma} \\
\dot{e}_{e m}=r_{e m}\left(-\mathrm{K}_{3} e_{e m}+\tilde{v}_{e m}\right) \\
\dot{\tilde{v}}_{e m}=-\mathrm{K}_{4} e_{e m}
\end{array}\right.
$$

Consider the candidate of Lyapunov function:

$$
V\left(e_{\Sigma}, \tilde{v}_{\Sigma}, e_{e m}, \tilde{v}_{e m}\right)=\frac{1}{2 r_{i m}} e_{\Sigma}{ }^{2}+\frac{1}{2 K_{2}} \tilde{v}_{\Sigma}{ }^{2}+\frac{1}{2 r_{e m}} e_{e m}{ }^{2}+\frac{1}{2 K_{4}} \tilde{v}_{e m}{ }^{2}
$$

Differential the Lyapunov function, the following equality can be obtained.

$$
\dot{V}=-K_{1} e_{\Sigma}^{2}-K_{3} e_{e m}{ }^{2}
$$

This follows Lyapunov stability of the error system.

Moreover, we can conclude:

$$
\begin{aligned}
& \dot{V}=0 \Leftrightarrow e_{\Sigma}=0, e_{e m}=0, \tilde{v}_{\Sigma}=0, \tilde{v}_{e m}=0 \\
& \Leftrightarrow e_{\Sigma}=0, e_{e m}=0, \Delta_{\Sigma}=\tilde{v}_{\Sigma}, \Delta_{e m}=v_{e m}
\end{aligned}
$$

By LaSalle's invariant set principle, $\left(\mathrm{e}_{\Sigma}, \tilde{\mathrm{v}}_{\Sigma}, \mathrm{e}_{\mathrm{em}}, \tilde{\mathrm{v}}_{\mathrm{em}}\right)$ will converge to the set $(0,0,0,0)$.

Finally, the nonlinear feedback control law can be designed as:

$$
\begin{gathered}
u_{t h r}=\phi_{t h r}{ }^{-1}\left(\dot{m}_{t w c}-\dot{m}_{f}-\mathrm{K}_{1} e_{\Sigma}-\mathrm{K}_{2} \int e_{\Sigma} d t\right) / G_{t h r}\left(p_{i m}\right) \\
u_{E G R}=\phi_{E G R}{ }^{-1}\left(\dot{m}_{e i}+\dot{m}_{f}-\dot{m}_{t w c}+\mathrm{K}_{3} e_{e m}+\mathrm{K}_{4} \int e_{e m} d t\right) / G_{E G R}\left(p_{e m}, p_{i m}\right)
\end{gathered}
$$

Remark: The nonlinear feedback controller in this paper is designed based on the mean value model of equation (1)-(2), in which the dynamic model is designed based on mean value pressure lumping in the manifolds and the pulsations of intake and exhaust manifold pressure are not considered. However, the pulsation of the intake and exhaust manifold pressure are very important for the accurate control of gasoline engine with EGR ${ }^{[24]}$, the model with considering the pulsation based controller design will be studied in the future research.

\section{Experimental validation}


In this section, the experimental validation results of the proposed control scheme are conducted. Experimental validations were implemented on a state-of-art gasoline engine test bench. In this test bench, a 1.8 L-L4 high efficiency engine is equipped, and the engine technical parameters are shown in tablel.

Table 1 Main engine specifications

\begin{tabular}{|l|l|}
\hline 2ZR-FXE Engine & Details \\
\hline Cylinder arrangement & Inline 4-cylinders \\
\hline Displacements & 1797 CC \\
\hline Fuel system & Port injection \\
\hline Compression ratio & $13: 1$ \\
\hline Maximum power & $71 \mathrm{kw} @ 5200 \mathrm{rpm}$ \\
\hline Maximum torque & 142 N.m @ 3600 rpm \\
\hline Air system & EGR / Intake VVT \\
\hline
\end{tabular}

Figure 4 shows the configuration of the test bench, in which the engine is connected with a low-inertia alternating-current (AC) electrical dynamometer from horiba. The dynamometer can emulate the external load torque in real time. Kistler 6052CU20 cylinder pressure sensors are equipped in each cylinder of the engine, and the encoder of 2361B1 connected with the crankshaft. Here, we only focus on the cylinder 4 , the signals from the cylinder pressure sensor and encoder are processed by a charger amplifier of Kister $6054 \mathrm{C} 12$ before which are provided to the real time control system of dSPACE.

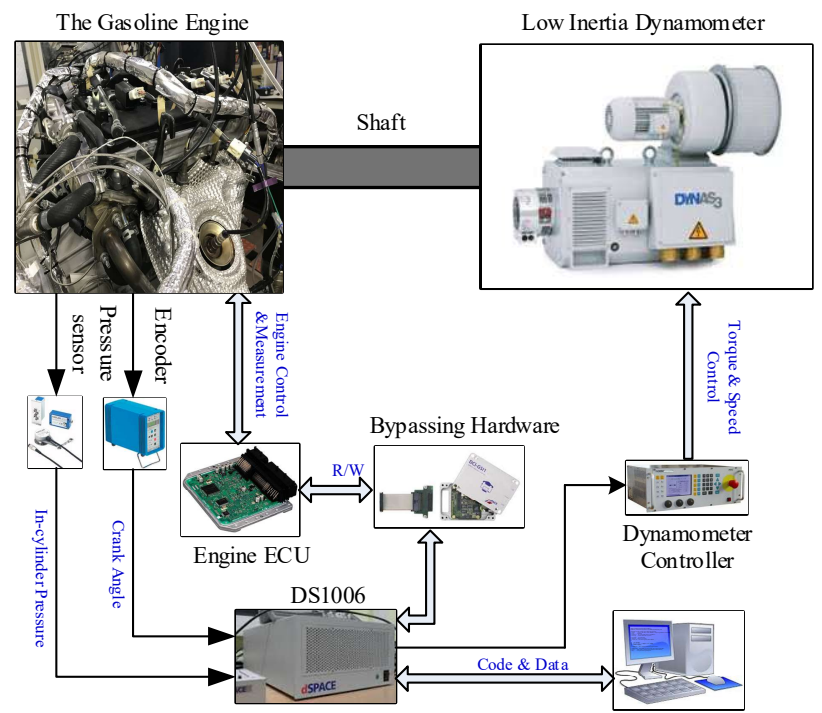

Fig. 4 Configuration of experimental setup

The real time control system dSPACE 1006 online processes the pressure signals to calculate the combustion parameters like IMEP, CA50, thermal efficiency and so on via the MATLAB/Simulink programming. On the another hand, the engine prototype ECU is equipped to fully control of the engine via the bypass connection. Hence, all the control algorithms can be programmed in the prototype controller, and the control program can be compiled and download to overwrite the bypass ECU to achieve the fully control of the engine.

\subsection{Model validation}

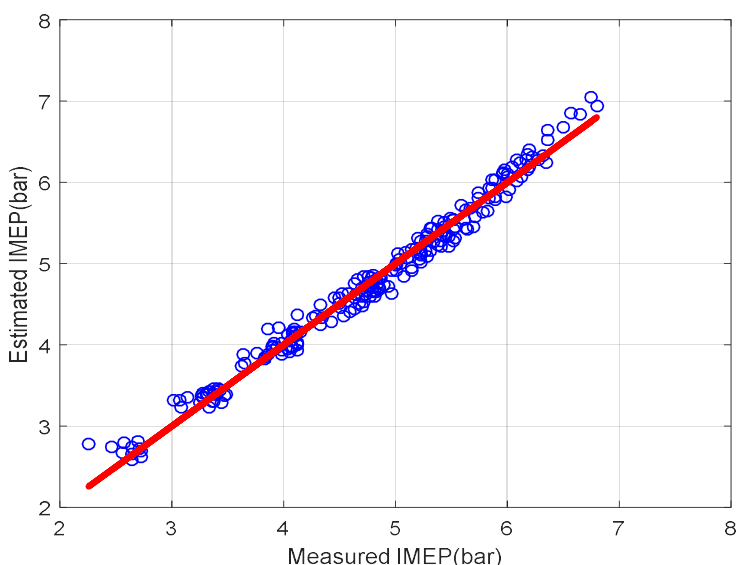

Fig. 5 the IMEP estimation results validation

The model of $\mu_{I M E P}$ in equation (5) is identified by experimental data at different conditions and the validation results are shown in figure 5. It demonstrates that the regression model of $\mu_{I M E P}$ can estimate IMEP quite well during different static conditions.

\subsection{CCO based set-point generation}

As discussed above, the optimal equilibrium points $\left(p_{t m}^{*}, p_{e m}^{*}, u_{t h r}^{*}, u_{E G R}^{*}\right)$ corresponding to the engine operating condition can be obtained by solving the chance-constraint optimization problem $\left(\mathcal{P}_{C C O}\right)$. Here, the probability $\beta$ is selected as 0.01 . Then, according to the proposition 1 , the constraint sampling number is selected as $\mathrm{N}=47$ which have confidence that the optimal solution of the scenario problem satisfies original chance-constraint problem with probability no smaller than 0.99 . (Here, $\mathrm{n}_{\mathrm{u}}$ is 2 . Then, the sampling number $\operatorname{Ngen}(\alpha ; \beta)=\mathrm{N}_{\text {gen }}(0.317$; 0.01 ) is 46.9896.)

As a comparison, another MAP is obtained by solving the following stationary optimization (SO) problem without IMEP chance-constraint:

$$
\begin{aligned}
\mathcal{P}_{S O}: & \min _{\left\{p_{i m}^{*}, p_{e m}^{*}, u_{t h r}^{*}, u_{E G R}^{*}\right\}}-\eta_{\mathrm{p}}\left(p_{i m}, p_{e m}\right) \\
\text { s.t. } & \tau_{d}-\tau_{e}\left(u_{E G R}, p_{i m}, p_{e m}, N_{e}\right)=0 \\
& \left(p_{i m}, p_{e m}\right) \in\left[\underline{p}_{i m}, \bar{p}_{i m}\right] \times\left[\underline{p}_{e m}, \bar{p}_{e m}\right] \\
& \left(u_{t h r}, u_{E G R}\right) \in\left[\underline{u}_{t i r}, \bar{u}_{t i r}\right] \times\left[\underline{u}_{E G R}, \bar{u}_{E G R}\right]
\end{aligned}
$$

The optimal problem formulated in (8) and (29) is solved by the SQP ${ }^{(25)}$ algorithm by matlab offline. The results of the optimal equilibrium points $\left(\mu_{t h r}^{*}, \mu_{E G R}^{*}, p_{i m}^{*}, p_{e m}^{*}\right)$ are obtained and shown in Fig.6. The corresponding constraints used in the optimal problem in $\mathcal{P}_{S O}$ and $\mathcal{P}_{S O}$ are $p_{\text {im }} \in\left[0,1.01 \times 10^{5}\right], \quad p_{\text {em }} \in$ $\left[0.98 \times 10^{5}, 2 \times 10^{5}\right], \mu_{t h r} \in[1.5,50]$, and $\mu_{E G R} \in[0,60]$. 

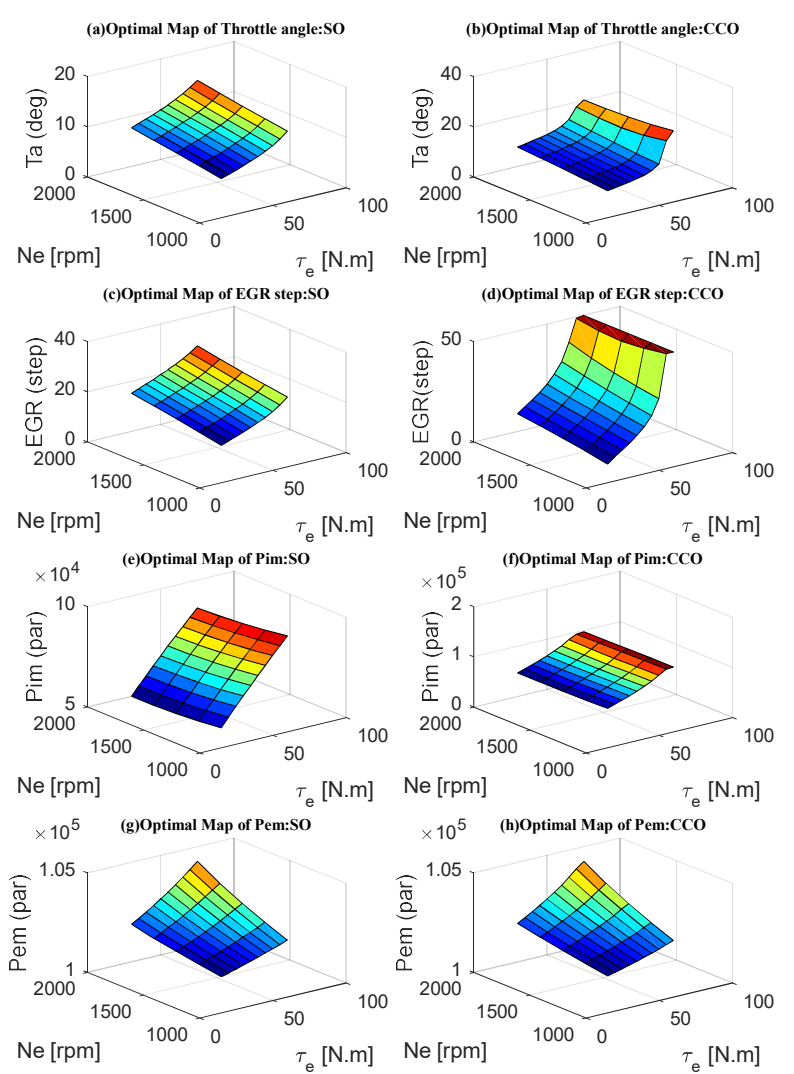

[N.m] $\mathrm{Ne}[\mathrm{rpm}] \quad \begin{array}{llll}1000 & 0 & \tau_{\mathrm{e}}[\mathrm{N} . \mathrm{m}]\end{array}$

Fig. 6 Set point MAP by solving stationary optimization problem and chance constraint optimization problem during different operating conditions.

\subsection{Experimental analysis of the control scheme}

Finally, the experimental validation was conducted to evaluate the effectiveness of the proposed control scheme. The experiments were conducted in the constant speed mode of dynamometer. Since we only focus on the EGR and throttle coordinating control of the proposed control scheme. The other engine conditions are controlled as followings, the water and oil temperature are controlled at a constant condition which are achieved by a temperature regulator, while the spark advance and the Lambda are controlled by the by-pass ECU to the MBT and stoichiometric mode according the engine conditions. To be mentioned that the exhaust manifold pressure state of the feedback variable is substituted by the exhaust manifold pressure estimator which is proposed in literature (22).

For a given torque reference of the dot blue line as shown in the subfigure (c) in Fig. 7, the optimal set-point block of control scheme in Fig. 3 gives an optimal reference $\mathrm{p}_{i m}^{*}$ and $\mathrm{p}_{e m}^{*}$ as shown in subfigure (a) and (b) respectively, here the dot blue line and the solid blue line stands for the reference obtained by the stationary optimization problem and chance constraint optimization problem respectively. Then, the designed nonlinear feedback controller of equation (28) and (29) calculate the control value of throttle valve angle and EGR valve angle to track the optimal reference $\mathrm{p}_{i m}^{*}$ and $\mathrm{p}_{e m}^{*}$. The corresponding control input of throttle valve and EGR valve of the experimental results are shown in the subfigure (f) and (g) respectively, and the $\mathrm{p}_{i m}$ and $\mathrm{p}_{\text {em }}$ can be tracked to the reference accurately which are exhibited in the subfigure (a) and (b).
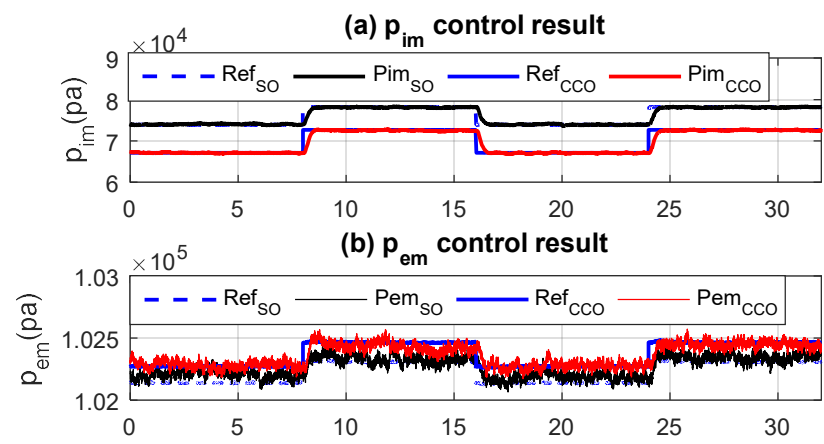

(c) Troque control result
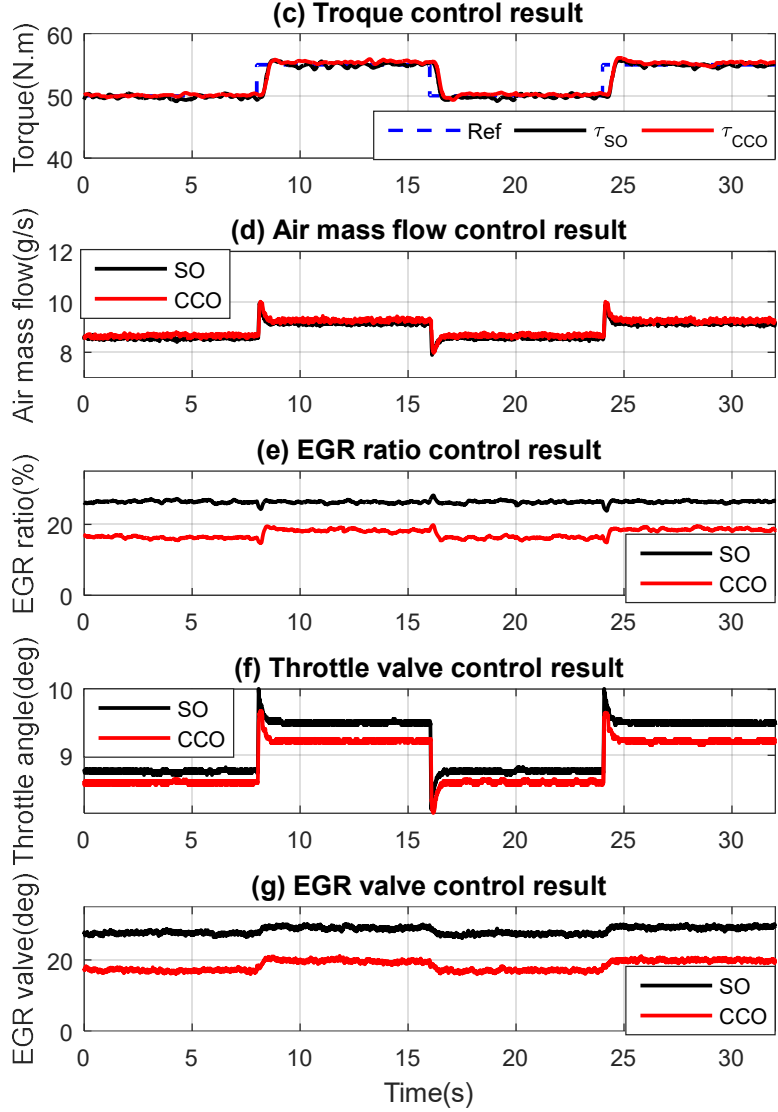

Fig. 7 the validation results of the controller

What's more, the air mass flow through the throttle valve can be controlled as almost the same of the two controller through the the $\mathrm{p}_{\text {im }}$ and $\mathrm{p}_{\text {em }}$ tracking which is illustrated in subfigure (d). To restrain of the IMEP probability constraint, the CCO based controller achieves lower intake manifold pressure but higher exhaust manifold pressure by controlling smaller EGR angle and throttle angle compared to SO based controller. Also, the EGR ratio of the $\mathrm{CCO}$ based control strategy is constrained to be smaller. Figure 8 further compares the corresponding combustion performance of the two controller, although the SO based control 
strategy achieves less pumping loss by using larger EGR ratio, the CCO based controller can obtain more stable combustion with a smaller variation of IMEP and CA50 by the constraint of the IMEP probability distribution. As a result, compared to $\mathrm{CO}$ based controller, the $\mathrm{CCO}$ based controller can reduce the $C O V_{I M E P}$ by $29.10 \%$ and $21.51 \%$ at condition of $50 \mathrm{~N} . \mathrm{m}$ and $55 \mathrm{~N} . \mathrm{m}$ respectively, while the brake efficiency of the both controller can keep as almost the same.
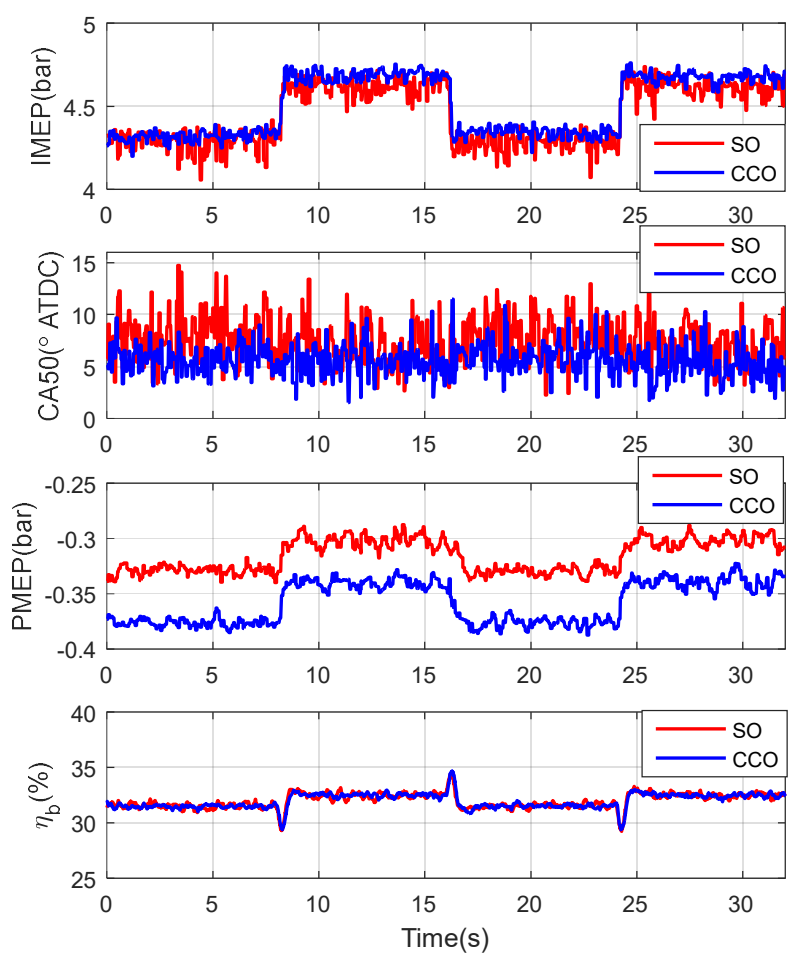

Fig. 8 the combustion results of the controller

Both the CCO based controller and the SO based controller can regulate the system states $p_{i m}$ and $p_{\text {em }}$ to the desired value during both steady condition and the transient conditions by the nonlinear feedback tracking controller, and the minimum/ maximum tracking error of $p_{i m}$ and $p_{e m}$ are $0.01 \mathrm{pa} / 463.55 \mathrm{pa}$ and $0.01 \mathrm{pa} / 195 \mathrm{pa}$ during the steady conditions. Then, the torque can also converge to the demand requirement and minimum/ maximum tracking error of torque is less than $0.01 \mathrm{~N} . \mathrm{m}$ and $1 \mathrm{~N} . \mathrm{m}$ at steady conditions. For transient condition at both the tip-in and tip-out conditions as shown in the subfigure (c) of figure 9, the $p_{i m}$ and $p_{e m}$ can be regulated to the reference within $0.5 \mathrm{~s}$ and the torque can be regulated to the reference within $0.55 \mathrm{~s}$. This means that the proposed stabilization nonlinear feedback controller of proposition 2 can achieve the stabilization equilibrium points tracking control no matter what the required mode it is. It can be concluded that the CCO based control strategy can achieve a good torque tracking control by guaranteeing a stable combustion. The Lyapunov based control design can guarantee the stability of the close loop dynamic system and achieve a good transient tracking performance.

\section{Conclusion}

In this paper, the IMEP probability constrained optimization method is proposed to restrain the combustion variation problem of EGR control, which is formulated by transforming the coefficient of variation into the IMEP probability constraint problem. Then, the optimal equilibrium points are designed by solving the formulated chance-constraint optimization problem with a scenario approach. Furthermore, the Lyapunov based nonlinear feedback control is designed to achieve the set points tracking control. The experimental control results shows that the proposed control scheme can guarantee a stable combustion while achieve fast transient regulation control of engine with guaranteeing of the asymptotic stability of the close loop system. The method of efficiency optimization under IMEP probability constraint can reduce the variation of combustion, which is an application of stochastic optimization in gasoline engine control.

Since the present approach is limited to mean value model based control, future research will focus on the expansion of the control design with considering intake and exhaust manifold pressure pulsation.

$\begin{array}{cc}\text { CA50 } & \text { The crank angle at } 50 \% \text { of heat release } \\ \text { CCO } & \text { Chance constraint optimization } \\ \text { ECU } & \text { Electronic control unit } \\ \text { EGR } & \text { Exhaust gas recirculation } \\ \text { IMEP } & \text { Indicated mean effective pressure } \\ \text { MBT } & \text { Maximum brake torque } \\ \text { LHV } & \text { Low heat value of fuel } \\ p_{i m} & \text { Intake manifold pressure } \\ p_{e m} & \text { Exhaust manifold pressure } \\ \text { PMEP } & \text { Pumping mean effective pressure } \\ \mathrm{r}_{\mathrm{e}} & \text { Compression ratio } \\ \text { Rim } & \text { Gas constant of intake manifold charge } \\ \text { Rem } & \text { Gas constant of exhaust manifold charge } \\ \text { SO } & \text { Stationary optimization } \\ \text { Ta } & \text { Throttle angle } \\ \text { Tim } & \text { Intake manifold charge temperature } \\ \text { Tem } & \text { Exhaust manifold charge temperature } \\ \text { TWC } & \text { Three way catalyst } \\ \mathrm{V}_{\mathrm{d}} & \text { Displacement } \\ \text { Vim } & \text { Intake manifold volume } \\ \text { Vem } & \text { Exhaust manifold volume } \\ \text { VVT } & \text { Variable valve timing } \\ m_{E G R} & \text { Mass flow through EGR valve } \\ m_{e i} & \text { Mass flow into in-cylinder } \\ & \end{array}$




$\begin{array}{cc}m_{t w c} & \text { Mass flow through TWC } \\ m_{t h r} & \text { Mass flow through throttle } \\ x_{E G R} & \text { EGR ratio } \\ \tau_{e} & \text { Torque } \\ \gamma & \text { Specific heat ratio } \\ & \text { Acknowledge }\end{array}$

The authors would like to acknowledge the Toyota Motor Corporation for the supports and help in this research.

This paper is written based on a proceeding presented at JSAE 2019 Annual Congress on October 17, 2018.

\section{References}

(1) Heywood J. B. Internal Combustion Engine Fundamentals. Mcgraw-hill, New York, 1988.

(2) Song D, Jia N, Guo X etc. Low pressure cooled EGR for improved fuel economy on a turbocharged PFI gasoline engine. No. 2014-01-1240. SAE Technical Paper, 2014.

(3) Alger T, Gingrich J, Roberts C and Mangold B. Cooled exhaust-gas recirculation for fuel economy and emissions improvement in gasoline engines. International Journal of Engine Research 12( 3): 252-264, 2011.

(4) Teodosio L, De Bellis V. and Bozza F. Fuel economy improvement and knock tendency reduction of a downsized turbocharged engine at full load operations through a low-pressure EGR system. SAE International Journal of Engines, 8(4): 1508-1519, 2015.

(5) Liu F, Pfeiffer J. M, Caudle R, Marshall P and Olin P. Low pressure cooled EGR transient estimation and measurement for a turbocharged SI engine. SAE Technical Paper, No. 2016-01-0618, 2016.

(6) Van Nieuwstadt M. J, Kolmanovsky I. V, Moraal P. E, Stefanopoulou, A and Jankovic M. EGR-VGT control schemes: experimental comparison for a high-speed diesel engine. IEEE Control Systems ,20(3): 63-79, 2000.

(7) Jankovic, M and Kolmanovsky, I. Constructive Lyapunov control design for turbocharged diesel engines. IEEE Transactions on Control Systems Technology, 8(2): 288-299, 2000.

(8) Kim S, Choi S, and Jin H. Pressure Model Based Coordinated Control of VGT and Dual-loop EGR in a Diesel Engine Air-path System. Int. J. Autom. Technol., 17(16):193-203, 2016.

(9) Zhu Q, Prucka, R Prucka, M and Dourra H. A nonlinear model predictive control strategy with a disturbance observer for spark ignition engines with external EGR. SAE International Journal of Commercial Vehicles. 10(2017-01-0608: 360-372, 2017.

(10) Kang S, Xie H, Hao T. and Shen T. A Disturbance Rejection-based Control Framework for SI-CAI Hybrid Combustion in Gasoline Engines. IFAC-PapersOnLine,
49(11): 665-672, 2016.

(11) Haskara I, Zhu G. and Winkelman J. Multivariable EGR/ spark timing control for IC engines via extremum seeking. In American Control Conference, Jun 14, 2006.

(12) Vance J.B., Singh A., Kaul B.C., Jagannathan S. and Drallmeier J.A. Neural network controller development and implementation for spark ignition engines with high EGR levels. IEEE transactions on neural networks, 18(4): 1083-1100, 2007.

(13) Rizzoni G. A stochastic model for the indicated pressure process and the dynamics of the internal combustion engine. IEEE Trans. Vehicular Technol. 38(3) : 180-192, 1989.

(14) Jones J.P, Frey J, Muske K, Scholl D. A cumulativesummation-based stochastic knock controller, Proc. Inst. Mech. Eng. D J. Automob. Eng. 224 (7) : 969-983, 2010.

(15) Wu, Y., Madan K., and Shen T. A stochastic logical system approach to model and optimal control of cyclic variation of residual gas fraction in combustion engines. Applied Thermal Engineering, 93: 251-259, 2016.

(16) Kall P, Wallace S. W, and Kall P. Stochastic programming. Chichester: Wiley, 1994.

(17) Ben-Tal A. and Nemirovski A. Robust convex optimization. Mathematics of operations research, 23(4): 769-805, 1998.

(18) Nemirovski A. Several NP-hard problems arising in robust stability analysis. Mathematics of Control, Signals and Systems, 6(2):99-105, 1993.

(19) Calafiore, G. C. and Campi M. C. The scenario approach to robust control design. IEEE Transactions on Automatic Control, 51(5): 742-753, 2006.

(20) Charnes A and Cooper W. W. Chance-constrained programming, Management Science, 6(1):73-89, 1959.

(21) Eriksson L and Nielsen L. Modeling and Control of Engines and Drivelines. John Wiley \& Sons, Sweden, 2014.

(22) Jiang W, Shen T. Lyapunov-Based Nonlinear Feedback Control Design for Exhaust Gas Recirculation Loop of Gasoline Engines. Journal of Dynamic Systems, Measurement, and Control, 141(5):051005, 2019.

(23) Zhang Y, Gao J, Shen T. Probabilistic Guaranteed Gradient Learning-Based Spark Advance Self-Optimizing Control for Spark-Ignited Engines. IEEE Transactions on Neural Networks and Learning Systems, 29(10):4683-4693, 2018.

(24 ) Kiwan R, Stefanopoulou, A.G, Martz J, Surnilla G, Ali I. and Styles D.J. Effects of differential pressure measurement characteristics on low pressure-EGR estimation error in Siengines. IFAC-PapersOnLine, 49(11): 722-729, 2016.

(25) Powell M. A Fast Algorithm for Nonlinearly Constrained Optimization Calculations. Numerical Analysis G. A. Watson, ed., Springer, Berlin, pp. 144-157, 1978. 